\title{
(6) OPEN ACCESS \\ Randomised masked clinical trial of the MGDRx eyebag for the treatment of meibomian gland dysfunction-related evaporative dry eye
}

\author{
Paramdeep Singh Bilkhu, Shehzad Anjam Naroo, James Stuart Wolffsohn
}

Ophthalmic Research Group, School of Life and Health Sciences, Aston University, Birmingham, West Midlands, UK

\section{Correspondence to} Professor James $\mathrm{S}$ Wolffsohn, School of Life and Health Sciences, Aston University, Birmingham B4 7ET, UK; j.s.w.wolffsohn@aston.ac.uk

Received 10 March 2014 Revised 16 June 2014 Accepted 22 June 2014 Published Online First 4 July 2014

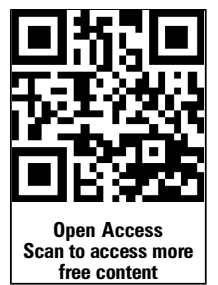

CrossMark

To cite: Bilkhu PS

Naroo SA, Wolffsohn JS. Br J Ophthalmol 2014;98: 1707-1711.

\section{ABSTRACT \\ Background/aims To investigate the efficacy and safety of the MGDRx EyeBag (The Eyebag Company, Halifax, UK) eyelid warming device. \\ Methods Twenty-five patients with confirmed} meibomian gland dysfunction (MGD)-related evaporative dry eye were enrolled into a randomised, single masked, contralateral clinical trial. Test eyes received a heated device; control eyes a non-heated device for 5 min twice a day for 2 weeks. Efficacy (ocular symptomology, noninvasive break-up time, lipid layer thickness, osmolarity, meibomian gland dropout and function) and safety (visual acuity, corneal topography, conjunctival hyperaemia and staining) measurements were taken at baseline and follow-up. Subsequent patient device usage and ocular comfort was ascertained at 6 months.

Results Differences between test and control eyes at baseline were not statistically significant for all measurements ( $p>0.05)$. After 2 weeks, statistically significant improvements occurred in all efficacy measurements in test eyes $(p<0.05)$. Visual acuity and corneal topography were unaffected ( $p>0.05$ ). All patients maintained higher ocular comfort after 6 months $(p<0.05)$, although the benefit was greater in those who continued usage 1-8 times a month $(p<0.001)$.

Conclusions The MGDRx EyeBag is a safe and effective device for the treatment of MGD-related evaporative dry eye. Subjective benefit lasts at least 6 months, aided by occasional retreatment.

Trial registration number NCT01870180.

\section{INTRODUCTION}

Eyelid warming therapy is considered the mainstay of treating meibomian gland dysfunction (MGD). ${ }^{1}$ A variety of devices have been developed and shown to be efficacious in the treatment of MGD, but many are not commercially available. ${ }^{2-6}$ More recent studies focus on in-office treatment procedures, and have found long-term improvements in MGD, ${ }^{7-10}$ but these may be reserved for severe cases or those unresponsive to conventional procedures.

There are no agreed-upon or standardised eyelid warming procedures in the scientific literature. Patient compliance issues relate to insufficient heating, duration and frequency of use. ${ }^{1}$ Studies which have attempted to optimise treatment using warm moist compresses suggest that precise and intense treatment is required to maintain sufficient eyelid warming, ${ }^{11}$ which would require high compliance and ability.
However, the MGDRx EyeBag (eyebag), a commercially available eyelid warming device, may allow for improved compliance owing to its simple design. A recent study found that it produced significant increases in tear film lipid layer thickness and break-up time in healthy subjects. ${ }^{12}$ Increases in temperature sufficient to melt meibum ${ }^{1}$ was also observed on the upper and lower internal and external eyelids, suggesting it may be effective in treating MGD and evaporative dry eye caused by an ineffective tear film lipid layer. ${ }^{12}$ The aim of this study was to therefore investigate the efficacy and safety of the eyebag in patients with MGD-related evaporative dry eye.

\section{MATERIALS AND METHODS}

The study was a randomised, contralateral (one-test eye, fellow eye control), examiner-masked clinical trial, received ethical approval from the Aston University Research Ethics Committee and was registered as a clinical trial (NCT01870180: ClinicalTrials.gov). The research was conducted in accordance with the principles expressed in the Declaration of Helsinki.

\section{Subjects}

Subjects were identified during routine eye examination in a local primary care optometry practice, and were required to be $\geq 18$ years old, with no systemic disease or medications known to affect the eyes, and no other active eye disease except symptomatic MGD-related evaporative dry eye. Diagnosis of at least mild severity was based upon recommendation by the diagnostic subcommittee of the International Workshop on Meibomian Gland Dysfunction: Ocular Surface Disease Index (OSDI) score of $>12$; presence of meibomian gland orifice plugging/obstruction on the lower or upper eyelids of both eyes; abnormal meibomian gland function in at least one eye $(\leq 20$ years old: quality or expressibility score $>1$; $>20$ years old: quality and expressibility score $\geq 1$; see below). ${ }^{13}$ Non-invasive tear film break-up time (NITBUT) $<10 \mathrm{~s}$ in at least one eye, and a negative Schirmer-1 test (>5.5 $\mathrm{mm}$ after $5 \mathrm{~min}$ ) in at least one eye to differentiate between evaporative and aqueous deficiency dry eye. ${ }^{13}$ Eligible subjects were enrolled with written informed consent and attended for a baseline visit (Day 0) where the following battery of tests were performed in sequence.

\section{Effectiveness measures}

Ocular symptomology: dry eye symptoms were assessed for each eye using the OSDI. 
Meibomian gland function: based upon meibum quality and expressibility using a slit-lamp. ${ }^{13}$ Quality and expressibility of meibum from the central 8 meibomian glands on the lower and upper eyelid was graded on a 4-point scale (quality: $0=$ clear fluid, $1=$ cloudy fluid, 2 =cloudy particulate fluid, $3=$ inspissated like toothpaste; expressibility: $0=$ all glands expressible, $1=3-4$ glands expressible, 2=1-2 glands expressible, $3=$ no glands expressible) following firm digital pressure to the eyelid margins. Scores from the upper and lower eyelids were added to give a composite value for quality and expressibility.

Meibomian gland dropout: measured via infrared meibography using the Keratograph $5 \mathrm{M}$ (Oculus Optikgeraete $\mathrm{GmbH}$, Wetzlar, Germany) for the central 15 meibomian glands in the upper and lower everted eyelids of each eye. Meibomian gland dropout was graded using a 4 -point scale $(1=$ no partial glands; $2 \leq 25 \%$ partial glands; $3=25-75 \%$ partial glands; $4 \geq 75 \%$ partial glands). A partial gland was defined as one that is incomplete (presumed relative to the length of neighbouring intact meibomian glands) and present in lumps or clusters. ${ }^{13}$ Scores from the upper and lower eyelids were added to give a composite value.

Tear film osmolarity: measured in each eye using the OcuSense TearLab (TearLab Corporation, San Diego, USA). Two measurements were taken in random order from each eye and were averaged to give a mean value for each eye. ${ }^{14}$

Tear film lipid layer thickness: measured non-invasively using a slit-lamp-mounted TearScope (Keeler, Windsor, UK) for each eye in random order. The thickness range of the tear film lipid layer was deduced from interference patterns observed using a standardised grading scale, where Grade $1=13-50 \mathrm{~nm}$; Grade $2=30-50 \mathrm{~nm}$; Grade 3=50-70 nm; Grade 4=80-90 nm; Grade $5=90-180 \mathrm{~nm}$; Grade $6 \geq 200 \mathrm{~nm}^{15}$

Tear film stability: was determined by assessing the NITBUT using a slit-lamp-mounted TearScope on each eye in random order. NITBUT was defined as the time between the last complete blink and the appearance of a break or distortion in the fine grid pattern. This was repeated two more times, and the values were averaged to give a mean value. ${ }^{15}$

Tear Film Meniscus Height (TMH): measured using the Keratograph $5 \mathrm{M}$. Images of the right and left eyes in primary gaze were captured immediately postblink. Digital callipers were used to measure the TMH located directly below the centre of the pupil, and was defined as the distance between the lower eyelid margin and the upper limit of the reflective zone-repeatability 95\% CI $\pm 0.06 \mathrm{~mm}$.

\section{Safety measures}

Visual acuity: best corrected monocular and binocular visual acuity was measured using a digital $\log$ MAR chart at $6 \mathrm{~m}$, randomised between presentations (Test Chart 2000; Thomson Software Solutions, London, UK). Visual acuity was determined using letter by letter scoring with each letter corresponding to $-0.02 \log$ MAR units.

Corneal topography: was measured on each eye using the Keratograph 5M.

Conjunctival hyperaemia: digital images of the nasal and temporal bulbar conjunctiva were captured and analysed to provide an objective measurement via edge detection and colour extraction. $^{16}$

Ocular surface staining: damage to the conjunctiva and cornea was assessed via instillation of lissamine green (GreenGlo, Sigma Pharmaceuticals, Monticello, USA) and fluorescein sodium (Fluorets, Chauvin Pharmaceuticals, London, UK) on each eye. Corneal, nasal and temporal bulbar conjunctival staining was graded individually using the Oxford system on a 6-point scale $(0-5)$ to provide a composite score $(0-15)$ for each eye. ${ }^{17}$

\section{Intervention}

Subjects were then randomised to receive a heated eyebag (40 s in microwave on full power) on either the left or right eye (test), chosen by random number generation. The contralateral eye received a non-heated eyebag (control). Subjects were instructed to use the eyebags at the same time twice a day (morning and evening, separated by at least $12 \mathrm{~h}$ ) for 2 weeks (Days 1-14) and were required to provide feedback-subjects were texted twice a day (morning and evening) to grade ocular comfort for each eye on a 1-10 scale immediately before applying the eyebags $(1=$ poor, $10=$ excellent $)$. This score was also obtained at baseline. The examiner was masked to which eye received the heat treatment. Subjects then attended for follow-up (Day 15) where the baseline measurements were repeated, and were left with the eyebags to maintain their treatment if they felt it beneficial. Subsequent patient usage and reported ocular comfort (1-10 scale) was ascertained at $6 \pm 1$ months.

\section{Statistics}

Statistical analysis was performed using SPSS for Microsoft Windows (Chicago, Illinois, USA). Differences between control and test eyes for visual acuity, ocular symptomology, corneal topography, conjunctival hyperaemia, and tear film stability, meniscus height, and osmolarity were evaluated by paired $t$ tests, where the normal distribution was confirmed (Kolmogorov-Smirnov test: $\mathrm{p}>0.05$ ). Differences between control and test eyes for non-normally distributed measures of tear film lipid layer thickness, meibomian gland function and dropout, and staining were evaluated by the Wilcoxon signed-rank test. Changes in ocular comfort scores over time were evaluated using Friedman's test, and posthoc analysis was performed using Wilcoxon signed-rank tests. To detect a primary treatment effect of 1 unit change in meibomian gland dropout $(\beta=0.2, \alpha=0.05), 23$ subjects were required as each subject acted as their own control based upon published data (SD in MGD patients $= \pm 1.7$ per eight glands). ${ }^{18}$ To account for possible drop-out, 25 patients (19 female, 6 male) were enrolled and completed the clinical trial (mean age: $28.7 \pm 7.8$ years; range $19-42$ years).

\section{RESULTS}

At baseline, there was no statistically significant difference in ocular comfort scores between control and test eyes (mean score control eye: $5.15 \pm 1.57$; test eye $5.25 \pm 1.33 ; \mathrm{Z}=-0.30$; $\mathrm{p}=0.76$ ). An improvement in ocular comfort scores over time was observed over the 2 week treatment period in test eyes $\left(\mathrm{X}^{2}=340.88, \mathrm{p}<0.001\right)$ but not control eyes $\left(\chi^{2}=27.78\right.$, $\mathrm{p}=0.42)$, with peak mean ocular comfort score in the test eye on the morning of Day $14(8.55 \pm 0.89)$. A statistically significant difference in comfort scores between control and test eyes occurred from the evening on Day 1 (control eye: 5.00 1.45 ; test eye: $6.40 \pm 1.35 ; Z=-3.72, p<0.001)$, and this difference remained statistically significant $(p<0.001)$ for the entire treatment period (figure 1).

The difference between test and control eyes at baseline was not statistically significant for all measurements $(p>0.05$; table 1). After the treatment period, there was a statistically significant improvement in all efficacy measurements in test eyes only $(p<0.05)$, except for meibomian gland expressibility in control eyes $(\mathrm{p}<0.05)$. Visual acuity and corneal topography 
Figure 1 Mean ocular comfort scores for control eyes and test eyes during the treatment period (Day 1 to Day 14). Error bars represent 1 SD. The difference in ocular comfort scores between control and test eyes was statistically significant $(p<0.001)$ from the evening of Day 1 onwards.

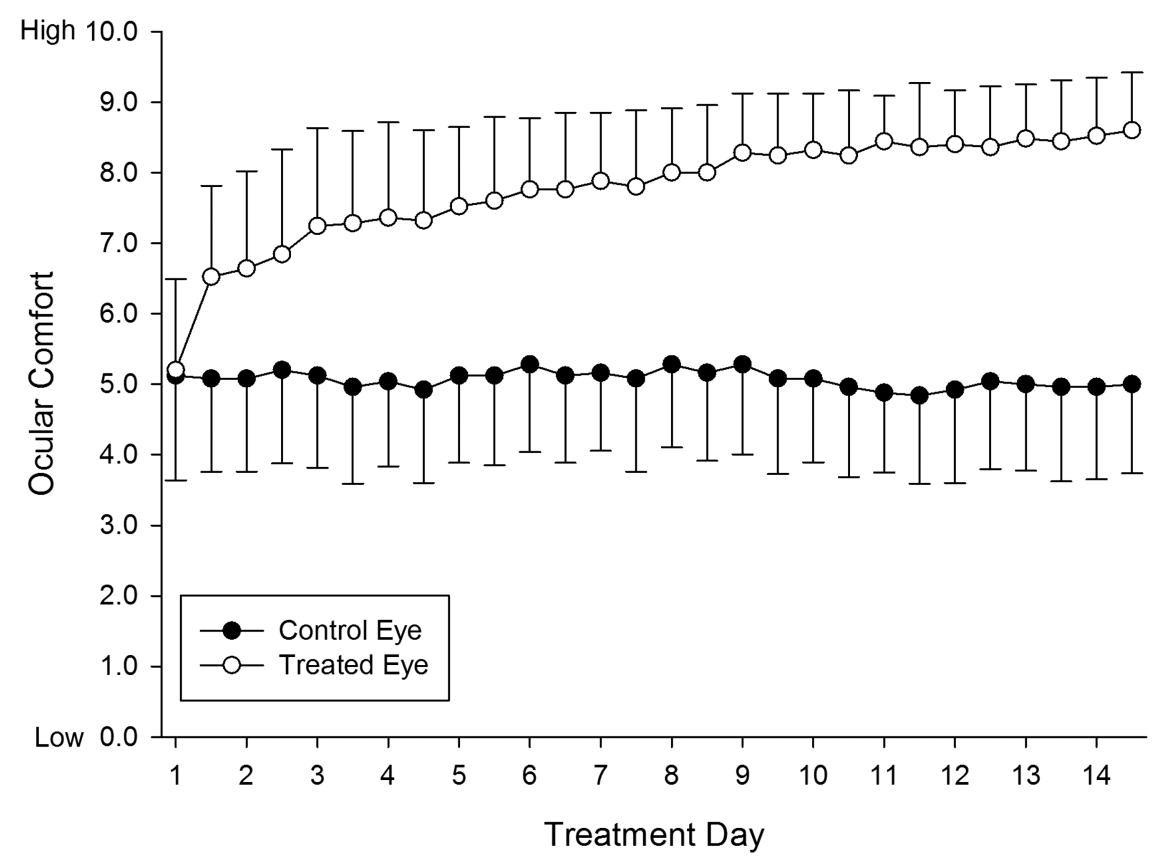

were unaffected $(\mathrm{p}>0.05)$, whereas conjunctival hyperaemia and ocular surface staining decreased in test eyes (table 1 ).

After 6 months, four patients reported having stopped using the eyebag, but their ocular comfort was still better than baseline $(6.20 \pm 1.03$ vs $4.80 \pm 1.40, p=0.039)$. Patients $(n=8)$ who continued to use the treatment for 1-4 weeks had a greater ocular comfort benefit $(7.79 \pm 0.89$ vs $5.64 \pm 1.33, \mathrm{p}<0.001)$, but the greatest scores were achieved by those who continued to use the eyebag $1-8$ times a month $(7.96 \pm 1.46$ vs $5.12 \pm 1.50$, $\mathrm{p}<0.001)$.

One patient reported a transient stinging sensation when the heated eyebag was placed on the upper eyelid on the first four occasions. No other adverse event was reported by this patient or the others for the duration of treatment period.

\section{DISCUSSION}

The results demonstrate statistically significant improvements in meibomian gland dropout, TMH, osmolarity, conjunctival hyperaemia and staining in test eyes only (table 1). Given that measurements were taken on two separate occasions, it was not possible to determine a natural history of the treatment approach. It is likely that the frequent and regular heating melted the abnormal meibum, clearing the obstruction within the meibomian gland, allowing the meibum to secrete on the eyelid margin. ${ }^{19}$ The increased quantity of meibum available may, therefore, help thicken and restore normal tear film lipid layer function such that evaporation of the underlying aqueous is prevented, tear film stability is improved, tear film osmolarity and ocular surface staining reduced and tear film meniscus height increased. ${ }^{20}$ This is supported by the significant positive correlation observed between lipid layer thickness and tear film stability. ${ }^{21}$ Furthermore, meibum quantity or evaporation rates have previously been shown to positively correlate with tear film stability. ${ }^{22}$

Indeed, NITBUT (by $1.9 \pm 1.3 \mathrm{~s}$ ) and tear film lipid layer thickness (by $1.1 \pm 0.8$ grades) demonstrated a statistically

Table 1 Mean $( \pm 1$ SD) values for all efficacy and safety parameters for test and control eyes at baseline and follow-up

\begin{tabular}{|c|c|c|c|c|c|c|c|}
\hline & \multicolumn{3}{|l|}{ Baseline } & \multicolumn{3}{|l|}{ Follow-up } & \multirow{2}{*}{$\begin{array}{l}\text { Test eye compared } \\
\text { to baseline }(p)\end{array}$} \\
\hline & Control & Test & Significance & Control & Test & Significance & \\
\hline OSDI & $43.0 \pm 14.4$ & $43.9 \pm 13.4$ & 0.36 & $39.8 \pm 12.7$ & $20.7 \pm 8.7$ & $<0.001^{*}$ & $<0.001^{*}$ \\
\hline Meibomian gland quality & $2.40 \pm 1.09$ & $2.35 \pm 0.99$ & 0.76 & $2.05 \pm 1.23$ & $1.3 \pm 0.92$ & $<0.05^{*}$ & $<0.001 *$ \\
\hline Meibomian gland expressibilty & $2.00 \pm 1.08$ & $2.15 \pm 0.89$ & 0.26 & $1.70 \pm 1.03$ & $1.35 \pm 0.82$ & $<0.05^{*}$ & $<0.001^{*}$ \\
\hline Meibomian gland drop-out & $2.80 \pm 1.24$ & $2.90 \pm 1.25$ & 0.41 & $2.65 \pm 1.04$ & $2.05 \pm 1.15$ & $<0.05^{*}$ & $<0.05^{*}$ \\
\hline Osmolarity (mOsm/L) & $310.3 \pm 10.6$ & $310.9 \pm 9.5$ & 0.43 & $308.9 \pm 11.6$ & $305.0 \pm 9.1$ & $<0.05^{*}$ & $<0.001^{*}$ \\
\hline Lipid layer thickness & $2.10 \pm 0.72$ & $2.05 \pm 0.89$ & 0.66 & $2.2 \pm 0.62$ & $3.1 \pm 0.91$ & $<0.05^{*}$ & $<0.001^{*}$ \\
\hline Tear film stability (s) & $9.29 \pm 3.64$ & $9.48 \pm 3.74$ & 0.12 & $9.32 \pm 3.56$ & $11.37 \pm 3.45$ & $<0.001^{*}$ & $<0.001^{*}$ \\
\hline Tear film meniscus height (mm) & $0.34 \pm 0.06$ & $0.34 \pm 0.07$ & 0.69 & $0.34 \pm 0.06$ & $0.36 \pm 0.06$ & $<0.001^{*}$ & $<0.05^{*}$ \\
\hline Visual acuity (LogMAR) & $-0.04 \pm 0.03$ & $-0.04 \pm 0.03$ & 0.79 & $-0.05 \pm 0.03$ & $-0.05 \pm 0.03$ & 0.42 & 0.06 \\
\hline Corneal topography (e) & $0.37 \pm 0.15$ & $0.37 \pm 0.16$ & 0.89 & $0.37 \pm 0.15$ & $0.37 \pm 0.17$ & 0.91 & 0.65 \\
\hline Conjunctival hyperaemia vessel coverage & $1.86 \pm 1.52$ & $1.61 \pm 1.19$ & 0.28 & $1.44 \pm 1.06$ & $0.94 \pm 0.65$ & $<0.05^{*}$ & $<0.05^{*}$ \\
\hline Conjunctival hyperaemia coloration & $38.81 \pm 1.62$ & $38.53 \pm 1.66$ & 0.23 & $38.49 \pm 1.42$ & $38.00 \pm 1.41$ & $<0.05^{*}$ & $<0.05^{*}$ \\
\hline Ocular surface staining & $3.2 \pm 2.06$ & $3.75 \pm 1.71$ & 0.07 & $2.95 \pm 1.39$ & $1.85 \pm 0.99$ & $<0.05^{*}$ & $<0.001^{*}$ \\
\hline
\end{tabular}


significant increase following heat treatment. Although a recent study investigating the effect of warm moist air goggles in MGD patients found no statistically significant increase in tear film stability 10 min after use, ${ }^{23}$ Olson et al (2003) found an $80 \%$ increase in lipid layer thickness in MGD-related dry eye patients after 5 min into treatment with warm moist compress heated to $40^{\circ} \mathrm{C}$ for $30 \mathrm{~min}$, and $66 \%$ increase $5 \mathrm{~min}$ after removal. ${ }^{3}$ Thus, it may be that improvements in tear film stability and lipid layer thickness in MGD patients can only be achieved where eyelid warming devices make contact with the eyelids.

Meibomian gland function also improved with the heated eyebag after the treatment period, where there were statistically significant improvements in meibum quality and expressibility in test eyes. However, the improvement in meibum quality compared to control eyes was only statistically significant between the lower eyelids. At baseline, meibum quality grade on the lower eyelid was significantly greater than the upper eyelid of test and control eyes, such that the upper eyelid was considered normal-thus, improvements in meibum quality may only have been detected in the lower eyelids owing to the limited resolution of the grading scale. Of interest was the statistically significant improvement in expressibility in control eyes (composite reduction of $0.35 \pm 0.81$ ). The non-heated eyebag may have warmed the eyelids by insulating and preventing heat loss, which subsequently brought about an improvement in the abnormal meibum. The frequent application of the eyebag with the hands may have also massaged the eyelids to help clear the obstructed glands. Since the heated and non-heated eyebags were applied at the same time and duration, this massaging effect may have contributed at least in part to the greater improvement in expressibility observed in test eyes (composite reduction of $0.80 \pm 0.70)$. With respect to the semiquantitative nature of the grading scale, eyelids with 5,6 , or 7 expressible glands were recorded as 'grade 1'-thus, the improvements observed may have been underestimated.

Furthermore, a statistically significant improvement in ocular symptoms was also observed in test eyes only. Surprisingly, the improvement in ocular comfort scores was observed as early as the evening of treatment Day 1, suggesting that the eyebag produces an increase in ocular comfort for at least $12 \mathrm{~h}$ following single use. Although subjects still had raised ocular comfort at around 6 months after the trial, comfort was maintained best in those who continued treatment $1-8$ times a month. It should be noted that subjects were not masked and, therefore, some placebo effect could be possible, although the effect was marked and occurred in all subjects.

Eyelid warming therapy associated with eyelid massage has been suggested to induce corneal deformation due to increases in corneal temperature, and with concurrent massaging, possible ectasia. ${ }^{24}$ Only one case of corneal deformation following such treatment has been reported, but the duration of treatment in this case was far longer (15 min twice a day for 7 weeks) than that prescribed herein. Transient visual degradation without changes in corneal topography has been observed after warm compresses $\left(45^{\circ} \mathrm{C}\right)$ were applied every $2 \mathrm{~min}$ for $30 \mathrm{~min}$ without massage, ${ }^{25}$ but again the duration of treatment and peak temperature was sustained for longer than the present study and the treatment method is not typically advised to MGD patients. Therefore, it appears that corneal deformation and/or visual changes, may only occur following unusually long and intense treatment application durations and long-term therapy associated with eyelid rubbing.

Given there was no reduction in visual acuity and no change in corneal topography from baseline after the treatment period, the risk of corneal deformation with the MGDRx EyeBag, as prescribed, appears to be minimal. Further, there was a statistically significant improvement in ocular surface staining (particularly the cornea) and conjunctival hyperaemia in test eyes. Thus, when used based upon the treatment regimen prescribed herein, the MGDRx EyeBag may be considered a safe and effective eyelid warming device with a low risk of corneal deformation and visual changes, and results in improved comfort and tear film parameters involved in evaporative dry eye in patients with MGD.

Acknowledgements We would like to thank Mr Kevin Blake FBDO CL for his very kind help in recruiting and screening the study patients.

Contributors All authors took an active part in the design, conduct, data analysis and publication drafting and approval.

Funding The MGDRx EyeBags were supplied at no cost by The EyeBag Company Ltd (Halifax, West Yorkshire, UK, HX3 OWY).

\section{Competing interests None.}

Patient consent Obtained.

Ethics approval Aston University Ethics Committee.

Provenance and peer review Not commissioned; externally peer reviewed.

Data sharing statement All data relating to the study is published in the manuscript and approached to share this can be made to the corresponding author.

Open Access This is an Open Access article distributed in accordance with the Creative Commons Attribution Non Commercial (CC BY-NC 4.0) license, which permits others to distribute, remix, adapt, build upon this work non-commercially, and license their derivative works on different terms, provided the original work is properly cited and the use is non-commercial. See: http://creativecommons.org/ licenses/by-nc/4.0/

\section{REFERENCES}

1 Geerling G, Tauber J, Baudouin C, et al. The international workshop on meibomian gland dysfunction: report of the subcommittee on management and treatment of meibomian gland dysfunction. Invest Ophthalmol Vis Sci 2011;52:2050-64.

2 Goto $\mathrm{E}$, Monden $\mathrm{Y}$, Takano $\mathrm{Y}$, et al. Treatment of non-inflamed obstructive meibomian gland dysfunction by an infrared warm compression device. $\mathrm{Br} J$ Ophthalmol 2002;86:1403-7.

3 Olson MC, Korb DR, Greiner JV. Increase in tear film lipid layer thickness following treatment with warm compresses in patients with meibomian gland dysfunction. Eye Contact Lens 2003;29:96-9.

4 Mori A, Shimazaki J, Shimmura S, et al. Disposable eyelid-warming device for the treatment of meibomian gland dysfunction. Jpn J Ophthalmol 2003;47:578-86.

5 Matsumoto Y, Dogru M, Goto E, et al. Efficacy of a new warm moist air device on tear functions of patients with simple meibomian gland dysfunction. Cornea 2006;25:644-50

6 Ishida R, Matsumoto Y, Onguchi T, et al. Tear Film with "Orgahexa EyeMasks" in Patients with Meibomian Gland Dysfunction. Optom Vis Sci 2008;85:684-91.

7 Friedland BR, Fleming CP, Blackie CA, et al. A novel thermodynamic treatment for meibomian gland dysfunction. Curr Eye Res 2011;6:79-87.

8 Greiner JV. A single LipiFlow Thermal Pulsation System treatment improves meibomian gland function and reduces dry eye symptoms for 9 months. Curr Eye Res 2012;37:272-8

9 Lane SS, DuBiner HB, Epstein RJ, et al. A new system, the LipiFlow, for the treatment of meibomian gland dysfunction. Cornea 2012;31:396-404.

10 Greiner JV. Long-term (12-month) improvement in meibomian gland function and reduced dry eye symptoms with a single thermal pulsation treatment. Clin Exp Ophthalmol 2013;41:524-30.

11 Blackie CA, Solomon JD, Greiner JV, et al. Inner eyelid surface temperature as a function of warm compress methodology. Optom Vis Sci 2008;85:675-83.

12 Bilkhu PS, Naroo SA, Wolffsohn JS. Effect of a commercially available warm compress on eyelid temperature and tear film in healthy eyes. Optom Vis Sci 2014:91:163-70.

13 Tomlinson A, Bron AJ, Korb DR, et al. The international workshop on meibomian gland dysfunction: report of the diagnosis subcommittee. Invest Ophthalmol Vis Sci 2011;52:2006-49

14 Tomlinson A, McCann LC, Pearce El. Comparison of human tear film osmolarity measured by electrical impedance and freezing point depression techniques. Cornea 2010;29:1036-41

15 Guillon JP. Non-invasive tearscope plus routine for contact lens fitting. Contact Lens Ant Eye 1998;21:31-40. 
16 Wolffsohn JS, Purslow C. Clinical monitoring of ocular physiology using digital image analysis. Contact Lens Ant Eye 2003;26:27-35.

17 Bron AJ, Evans VE, Smith JA. Grading of corneal and conjunctival staining in the context of other dry eye tests. Cornea 2003;22:640-50.

18 Mathers WD, Shields WJ, Sachdev MS, et al. Meibomian gland dysfunction in chronic blepharitis. Cornea 1991;10:277-85.

19 Knop E, Knop N, Millar T, et al. The international workshop on meibomian gland dysfunction: report of the subcommittee on anatomy, physiology, and pathophysiology of the meibomian gland. Invest Ophthalmol Vis Sci 2011;52:1938-78.

20 Foulks GN. The correlation between the tear film lipid layer and dry eye disease. Surv Ophthalmol 2007;52:369-74.
21 Isreb MA, Greiner JV, Korb DR, et al. Correlation of lipid layer thickness measurements with fluorescein tear film break-up time and Schirmer's test. Eye 2003;17:79-83.

22 Mathers W. Evaporation from the ocular surface. Exp Eye Res 2004;78 389-94.

23 Purslow $C$. Evaluation of the ocular tolerance of a novel eyelid-warming device used for meibomian gland dysfunction. Contact Lens Ant Eye 2013;36:226-31.

24 McMonnies CW, Korb DR, Blackie CA. The role of heat in rubbing and massage-related corneal deformation. Contact Lens Ant Eye 2012;35:148-54.

25 Solomon JD, Case CL, Greiner JV, et al. Warm compress induced visual degradation and Fischer-Schweitzer polygonal reflex. Optom Vis Sci 2007;84:580-7. 